\title{
Kallmann Syndrome Phenotype in a Female Patient with CHARGE Syndrome and CHD7 Mutation
}

\author{
TSUTOMU OGATA, IKUMA FUJIWARA*, EISHIN OGAWA*, NAOKO SATO, TORU UDAKA** \\ AND KENJIRO KOSAKI** \\ Department of Endocrinology and Metabolism, National Research Institute for Child Health and Development, Tokyo 157-8535, \\ Japan \\ *Department of Pediatrics, Tohoku University School of Medicine, Sendai 980-0878, Japan \\ **Department of Pediatrics, Keio University School of Medicine, Tokyo 160-8582, Japan
}

\begin{abstract}
We report on a 14 7/12-year-old Japanese female patient with CHARGE syndrome and CHD7 mutation who also exhibited Kallmann syndrome (KS) phenotype. She had poor pubertal development and apparently impaired sense of smell. A GnRH test showed severely compromised responses of $\mathrm{LH}(<0.5 \rightarrow<0.5 \mathrm{IU} / \mathrm{L})$ and FSH $(<0.5 \rightarrow 1.2 \mathrm{IU} / \mathrm{L})$, and magnetic resonance imaging delineated hypoplastic olfactory bulbs. Mutation analysis revealed a heterozygous nonsense mutation at exon 33 of $C H D 7(7027 \mathrm{C}>\mathrm{T}, \mathrm{Q} 2343 \mathrm{X})$. The results provide further support for the notion that KS phenotype can be included in the phenotypic spectrum of CHARGE syndrome, and indicate that CHARGE syndrome with KS phenotype is caused by a $C H D 7$ mutation.
\end{abstract}

Key words: CHARGE syndrome, Kallmann syndrome, Phenotype, CHD7

(Endocrine Journal 53: 741-743, 2006)

CHARGE syndrome is a congenital malformation disorder named by the acronym of coloboma, heart disease, atresia choanae, retarded growth and/or development, genital anomalies, and ear abnormalities [1]. In addition to these core features, hypogonadotropic hypogonadism $(\mathrm{HH})$ and olfactory dysfunction characteristic of Kallmann syndrome (KS) [2] are often observed in CHARGE syndrome. Wheeler et al. [3] documented the invariable occurrence of $\mathrm{HH}$, and Chalouhi et al. [4] described the frequent presence of olfactory dysfunction in CHARGE syndrome. Furthermore, Pinto et al. [5] reported the frequent occurrence of both $\mathrm{HH}$ and abnormal olfactory bulb development in this syndrome.

In 2004, Vissers et al. [6] reported that CHARGE

Received: June 13, 2006

Accepted: July 7, 2006

Correspondence to: Dr. Tsutomu OGATA, Department of Endocrinology and Metabolism, National Research Institute for Child Health and Development, 2-10-1 Ohkura, Setagaya, Tokyo 1578535, Japan syndrome is primarily caused by heterozygous loss-offunction mutations of $\mathrm{CHD} 7$ (chromodomain helicase DNA-binding protein 7) on 8q12.1. Subsequently, mutation analysis has been performed for a large number of CHARGE syndrome patients, identifying $\mathrm{CHD} 7$ mutations in $60-70 \%$ of patients $[7,8]$. However, mutation analysis remains poor in CHARGE syndrome patients with demonstrable KS phenotype. Here, we report $C H D 7$ mutation in a patient with CHARGE syndrome who also presented with KS phenotype.

\section{Case Report}

This Japanese female patient was born to healthy non-consanguineous parents at 38 weeks of gestation with a birth weight of $2.94 \mathrm{~kg}(-0.5 \mathrm{SD})$, after an uncomplicated pregnancy and delivery. At birth, she was noticed to have coloboma of the iris and retina and external ear defects. There was no choanal atresia. She had feeding difficulties and showed failure to thrive. At six months of age, auscultation indicated cardiac 
murmur, and echocardiography delineated atrial and ventricular septal defects, patent ductus arteriosus, and right aortic arch. She showed poor response to sounds, and was found to have bilateral hearing impairment by the auditory brainstem response test (right 60-70 dB, left $>115 \mathrm{~dB}$ ). She needed nasal tube feeding until 2 $8 / 12$ years of age, and was unable to walk without support until 3 years of age. At 3 years of age, her developmental quotient was assessed as 64 by the WISC-R test. On the basis of the above findings, she was diagnosed as having CHARGE syndrome.

At 14 7/12 years of age, she was evaluated for endocrine function because of poor pubertal development (breast, Tanner stage 2; pubic hair, Tanner stage 3). Her height was $153.6 \mathrm{~cm}(-0.6 \mathrm{SD})$ and her weight $49.9 \mathrm{~kg}(-0.1 \mathrm{SD})$. Her bone age was assessed as 12.0 years. Basal serum estradiol was $<10 \mathrm{pg} / \mathrm{mL}$, and a GnRH test $\left(100 \mu \mathrm{g} / \mathrm{m}^{2}\right.$ bolus i.v.; blood sampling at $0,30,60,90$, and $120 \mathrm{~min}$ ) showed severely compromised responses of LH (baseline $<0.5 \mathrm{mIU} / \mathrm{mL}$ [normal range: $0.5-5.0 \mathrm{mIU} / \mathrm{mL}$ ] and peak $<0.5 \mathrm{mIU} / \mathrm{mL}$ [1.5-8.0 mIU/mL]) and FSH (baseline $<0.5 \mathrm{mIU} / \mathrm{mL}$ [0.8-4.4 mIU/mL] and peak $1.2 \mathrm{mIU} / \mathrm{mL}$ [8.3-20.0 $\mathrm{mIU} / \mathrm{mL}]$ ). Other endocrine functions were normal. Her karyotype was 46,XX in all the 20 lymphocytes examined. Furthermore, since the parents suspected impaired sense of smell in her daily life, magnetic resonance imaging was performed, revealing hypoplastic olfactory bulbs and left side dominant cerebral atrophy. The anterior pituitary appeared to be small for her age. Thus, she was assessed to have $\mathrm{HH}$ and hypoplastic olfactory bulbs characteristic of KS, and was placed on sex steroid supplementation therapy. She had no other KS features such as mirror movements, renal agenesis, cleft palate, and dental agenesis.

\section{Mutational Analysis of CHD7}

After obtaining an informed consent, mutation analysis of $C H D 7$ was performed as described previously [7]. In brief, leukocyte genomic DNA of the patient was PCR-amplified for all the coding exons 2-37 and their splice sites, and the PCR products were screened for a mutation by the denaturing high-performance liquid chromatography (DHPLC) using an automated instrument WAVE (Transgenomic, Omaha, NE). When abnormal heteroduplex patterns were detected, the corresponding PCR products were subjected to direct sequencing on an ABI PRISM 3100 autosequencer

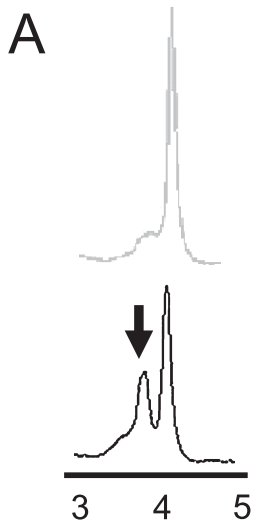

B
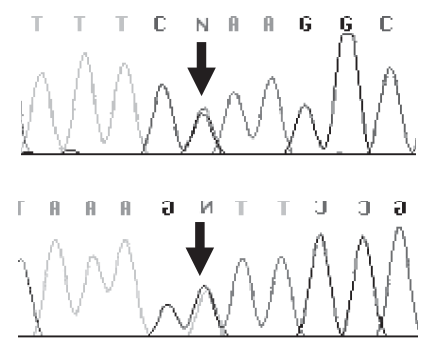

Fig. 1. Mutation analysis of $C H D 7$.

(A) Denaturing high-performance liquid chromatograms of the PCR products for exon 33 in a normal control (top) and the patient (bottom). The arrow indicates the abnormal chromatogram pattern found in the patient. The horizontal axis indicates the retention time (minute), and the vertical axis indicates the fluorescence intensity. (B) Automated sequencing of the PCR products for exon 33 in the patient. Chromatograms obtained with the forward primer (top) and the reverse primer (bottom) are shown. The arrows indicate the heterozygous nonsense mutation (7027C $>$ T, Q2343X).

(Perkin-Elmer, Foster City, CA).

DHPLC revealed an abnormal chromatogram for the PCR product of exon 33 in the patient (Fig. 1-A). Direct sequencing showed a heterozygous transition $7027 \mathrm{C}>\mathrm{T}$ resulting in a substitution of the 2343rd glutamine codon with a stop codon (Q2343X) (Fig. 1B). This mutation was absent in 50 normal subjects.

\section{Discussion}

This patient with CHARGE syndrome also had KS phenotype. The results provide further support for the notion that KS phenotype can be included in the phenotypic spectrum of CHARGE syndrome [3-5]. Furthermore, since a heterozygous nonsense mutation was identified in $\mathrm{CHD} 7$, this indicates that CHARGE syndrome with KS phenotype is caused by a $C H D 7$ mutation.

It remains to be clarified how a $C H D 7$ mutation leads to KS phenotype. It is notable, however, that the murine homolog Chd7 is clearly expressed in the olfactory epithelium [9] which is indispensable for the migration of $\mathrm{GnRH}$ and olfactory neurons into the brain and for the formation of olfactory bulbs $[2,10]$. 
Thus, a CHD7 mutation may primarily affect the development of olfactory epithelium, resulting in $\mathrm{HH}$ and olfactory dysfunction.

In summary, we observed KS phenotype in a patient with CHARGE syndrome and a $C H D 7$ mutation. Further studies will determine the phenotypic spectrum of CHD7 mutations as well as the molecular network involving $C H D 7$ and the previously known genes for KS, i.e., KAL1 (Kallmann syndrome 1) and FGFR1 (fibroblast growth factor receptor 1) [2].

\section{Acknowledgements}

This study was supported by grants for Child Health and Development (17C-2) and for Research on Children and Families from the Ministry of Health, Labor, and Welfare, and by a Grant-in-Aid for Scientific Research on Priority Areas from the Ministry of Education, Science, Sports and Culture (16086215).

\section{References}

1. Pagon RA, Graham JM Jr, Zonana J, Yong SL (1981) Coloboma, congenital heart disease, and choanal atresia with multiple anomalies: CHARGE association. $J$ Pediatr 99: 223-227.

2. Dodé C, Hardelin JP (2004) Kallmann syndrome: fibroblast growth factor signaling defect? $J \mathrm{Mol} \mathrm{Med}$ 82: 725-734.

3. Wheeler PG, Quigley CA, Sadeghi-Nejad A, Weaver DD (2000) Hypogonadism and CHARGE association. Am J Med Genet 94: 228-231.

4. Chalouhi C, Faulcon P, Le Bihan C, Hertz-Pannier L, Bonfils P, Abadie V (2005) Olfactory evaluation in children: application to the CHARGE syndrome. Pediatrics 116: e81-88.

5. Pinto G, Abadie V, Mesnage R, Blustajn J, Cabrol S, Amiel J, Hertz-Pannier L, Bertrand AM, Lyonnet S, Rappaport R, Netchine I (2005) CHARGE syndrome includes hypogonadotropic hypogonadism and abnormal olfactory bulb development. $J$ Clin Endocrinol Metab 90: 5621-5626.

6. Vissers LE, van Ravenswaaij CM, Admiraal R, Hurst JA, de Vries BB, Janssen IM, van der Vliet WA, Huys EH, de Jong PJ, Hamel BC, Schoenmakers EF, Brunner
HG, Veltman JA, van Kessel AG (2004) Mutations in a new member of the chromodomain gene family cause CHARGE syndrome. Nat Genet 36: 955-957.

7. Aramaki M, Udaka T, Kosaki R, Makita Y, Okamoto N, Yoshihashi H, Oki H, Nanao K, Moriyama N, Oku S, Hasegawa T, Takahashi T, Fukushima Y, Kawame H, Kosaki K (2006) Phenotypic spectrum of CHARGE syndrome with CHD7 mutations. J Pediatr 148: 410414.

8. Lalani SR, Safiullah AM, Fernbach SD, Harutyunyan KG, Thaller C, Peterson LE, McPherson JD, Gibbs RA, White LD, Hefner M, Davenport SL, Graham JM, Bacino CA, Glass NL, Towbin JA, Craigen WJ, Neish SR, Lin AE, Belmont JW (2006) Spectrum of CHD7 mutations in 110 individuals with CHARGE syndrome and genotype-phenotype correlation. Am J Hum Genet 78: 303-314.

9. Bosman EA, Penn AC, Ambrose JC, Kettleborough R, Stemple DL, Steel KP (2005) Multiple mutations in mouse Chd7 provide models for CHARGE syndrome. Hum Mol Genet 14: 3463-3476.

10. Rugarli EI, Ballabio A (1993) Kallmann syndrome: from genetics to neurobiology. JAMA 270: 2713-2716. 